\title{
Expression of CD24 in cholangiocarcinoma cells is associated with disease progression and reduced patient survival
}

\author{
SIRIPORN KEERATICHAMROEN ${ }^{1,2}$, KAWIN LEELAWAT $^{3,4}$, TAWEESAK THONGTAWEE $^{3}$, \\ SIRILUCK NARONG ${ }^{3}$, UMAAD AEGEM $^{3}$, SUPATHIP TUJINDA $^{3}$, \\ NIPHON PRADITPHOL ${ }^{3}$ and RUTAIWAN TOHTONG ${ }^{5}$ \\ ${ }^{1}$ Department of Molecular Medicine, Faculty of Science, Mahidol University, Bangkok 10400; ${ }^{2}$ Biochemistry Laboratory, \\ Chulabhorn Research Institute, Bangkok 10210; ${ }^{3}$ Department of Surgery, Rajavithi Hospital; ${ }^{4}$ College of Medicine, \\ Rangsit University; ${ }^{5}$ Department of Biochemistry, Faculty of Science, Mahidol University, Bangkok 10400, Thailand
}

Received March 18, 2011; Accepted May 12, 2011

DOI: $10.3892 /$ ijo.2011.1088

\begin{abstract}
Cholangiocarcinoma is frequently found to invade local tissues and metastasize to distal organs. We investigated the expression of CD24 in cholangiocarcinoma samples and its prognostic significance. In addition, the cellular function of CD24 was studied in the RMCCA1 cholangiocarcinoma cell line. High CD24 expression significantly correlated with lymph node metastasis and positive surgical margins in cholangiocarcinoma patients. Univariate and multivariate analyses further demonstrated that CD24 expression was significantly associated with the overall survival of these patients $(\mathrm{p}=0.007$ and $\mathrm{p}=0.040$, respectively). For in vitro studies, the magnetic-activated cell sorting (MACS) system was used to isolate $\mathrm{CD} 24^{+}$and $\mathrm{CD} 24^{-}$cell populations from RMCCA1 cells. CD24+ RMCCA1 cells had increased chemoresistance, adhesion $(\mathrm{p}=0.004)$, motility $(\mathrm{p}<0.001)$, migration $(\mathrm{p}<0.001)$ and invasion $(\mathrm{p}<0.001)$ capabilities when compared to CD24- cells. The matrix metalloproteinase (MMP)-7 was significantly elevated in $\mathrm{CD} 24^{+} \mathrm{RMCCA} 1$ cells $(\mathrm{p}=0.01)$. We found that inhibition of CD24 using siRNA silencing significantly decreased the invasive capacity of RMCCA1 cells. Both clinical and in vitro studies suggest that expression of CD24 is associated with cholangiocarcinoma disease progression. CD24 may thus serve as a new target for directed molecular therapy of cholangiocarcinoma.
\end{abstract}

\section{Introduction}

Cholangiocarcinoma is one of the most aggressive malignant tumors associated with local tissue invasion and a high rate of

Correspondence to: Dr Kawin Leelawat, Department of Surgery, Rajavithi Hospital, Bangkok 10400, Thailand

E-mail: kawin.leelawat@gmail.com

Abbreviations: DAPI, 4,6-diamidino-2-phenylindole dihydrochloride; ECM, extracellular matrix; FBS, fetal bovine serum; MACS, magnetic-activated cell sorting; MMP, matrix metalloproteinase

Key words: CD24, cholangiocarcinoma, invasion, metastasis, survival metastasis and is one of the most common causes of cancer death in Thailand (1). Three-year survival rates of $35-50 \%$ are achieved only in a subset of patients who have negative histological margins at the time of surgery (2-5). Palliative therapeutic approaches, including percutaneous and endoscopic biliary drainage, have generally been used for these patients because there is no effective chemotherapeutic treatment for cholangiocarcinoma. Therefore, identification of the molecules involved in cholangiocarcinoma cell progression is crucial for the development of novel drug treatments for this disease.

CD24 is a small, heavily glycosylated mucin-like glycosylphosphatidylinositol (GPI)-linked cell surface protein that serves as a ligand for P-selectin, an adhesion receptor on activated endothelial cells and platelets (6). CD24 can be detected in granulocytes, pre-B-cells and keratinocytes (7) and its expression in non-small cell lung cancer, ovarian cancer and hepatocellular carcinoma is associated with poor prognoses $(8,9)$. However, only limited studies have been published on CD24 expression in cholangiocarcinoma $(10,11)$ and there are no data on the mechanism of CD24 involvement in cholangiocarcinoma cell progression. In the present study, we investigated the clinicopathological significance of CD24 expression in human cholangiocarcinoma samples. We also determined the cellular function of CD24 in cholangiocarcinoma cells by isolating CD24+ and CD24- cells from the RMCCA1 cholangiocarcinoma cell line and assessing cell proliferation, chemoresistance and invasiveness of $\mathrm{CD} 24^{+}$versus CD24- cells.

\section{Materials and methods}

Human cholangiocarcinoma tissue samples. Cholangiocarcinoma tissue samples were obtained from cholangiocarcinoma patients who underwent a surgical resection at Rajavithi Hospital in Bangkok, Thailand from 2007-2009. The study was approved by the ethics committee of Rajavithi Hospital.

Immunohistochemical staining. Paraffin wax sections of cholangiocarcinoma specimens were dewaxed in xylene and transferred to alcohol. Endogenous peroxidase activity was blocked with $0.5 \%$ hydrogen peroxide in methanol, and the sections were boiled in $10 \mathrm{mM}$ citrate buffer ( $\mathrm{pH}$ 6.0) in a microwave oven 
(750 W) for antigen retrieval. Non-specific binding was blocked by incubation with $3 \%$ normal horse serum for $20 \mathrm{~min}$. Sections were incubated overnight at $4^{\circ} \mathrm{C}$ with a 1:1000 dilution of a mouse anti-CD24 monoclonal antibody. Biotinylated rabbit antimouse IgM (Dako, Glostrup, Denmark) was then added followed by an avidin-biotin-peroxidase conjugate (ABC Elite; Vector Laboratories, Burlingame, CA, USA) for $30 \mathrm{~min}$ at room temperature. The immunohistochemical reaction was developed with freshly prepared reagents from a Histofine SAB-PO kit (Nichirei Inc., Tokyo, Japan). Sections were then visualized under high power magnification (x400) using an Olympus BH2 microscope (field width, $0.5 \mathrm{~mm}$ ) and scored into one of the following three categories based on the percentage of positively stained cells: i) negative, $<5 \%$, ii) low, $5-50 \%$ and iii) high, $>50 \%$.

Cell culture. The human cholangiocarcinoma cancer cell line, RMCCA1 (12), was grown in Ham's F-12 medium (Invitrogen Life Technologies, Carlsbad, CA) supplemented with $10 \%$ FBS (fetal bovine serum) and $1 \%$ penicillin/streptomycin. In all experiments, cells were maintained at $37^{\circ} \mathrm{C}$ in a humidified $5 \% \mathrm{CO}_{2}$ incubator.

Immunofluorescence study. To examine CD24 protein expression, cells were plated and grown on glass coverslips. After fixation with $4 \%$ paraformaldehyde for $15 \mathrm{~min}$ at room temperature, cells were treated with $4 \%$ bovine serum albumin. Cells were then stained with a mouse anti-CD24 antibody (mouse monoclonal IgG; Abcam Inc., Cambridge, MA) followed by an Alexa Fluor 594 goat anti-mouse IgG secondary antibody (1:200; Molecular Probes, Eugene, Oregon). The cells were stained with DAPI (4, 6-diamidino-2-phenylindole dihydrochloride) (Invitrogen) and viewed under a fluorescence microscope.

Isolation of $\mathrm{CD}^{2} 4^{+}$and $\mathrm{CD} 24^{-}$populations by magnetic cell sorting. For magnetic cell sorting, RMCCA1 cells were incubated with a FITC-conjugated anti-human CD24 antibody (Miltenyi Biotec, Auburn, CA), magnetically labeled with antiFITC microbeads and then separated on a MACS MS column (Miltenyi Biotec) according to the manufacturer's instructions. The magnetic separation step was repeated twice to obtain high purity of the cell populations. The CD24+ and CD24- sorted cells were then evaluated for the efficiency of the magnetic separation by flow cytometry.

Cell proliferation assay. For proliferation assays, cells were seeded into 96-well culture plates at a density of 1,000 cells per well. Cells were incubated for 0-9 days before applying the WST-1 cell proliferation assay reagent (Roche Diagnostics, Laval, Quebec, Canada) according to the recommendations of the manufacturer. The degree of cell proliferation was assessed by determining the A450 $\mathrm{nm}$ of the cell culture medium after addition of WST-1 for $2 \mathrm{~h}$.

For cancer chemoresistance studies, 10,000 cells were treated with vehicle (normal medium) or $1-125 \mu \mathrm{g} / \mathrm{ml}$ cisplatin (Fresenius Kabi Oncology Ltd., Solan, India). Cells were then incubated for $48 \mathrm{~h}$ before applying WST-1. The number of viable cells after drug treatment was assessed using the WST-1 cell proliferation assay as described above. The drug concentration that resulted in a $50 \%$ decrease in relative absorbance was defined as the $\mathrm{IC}_{50}$.
In vitro adhesion assay. Cells were seeded into 96-well culture plates coated with Matrigel at a density of 10,000 cells per well. Cells were allowed to adhere for $30 \mathrm{~min}$. The nonadherent cells and media were then discarded, and the plates were washed with PBS. The number of adherent cells was assessed using WST-1.

Cell motility (wound-healing) assay. To assess cell motility, a wound-healing assay was performed followed by time-lapse live cell imaging. Wounds were generated by puncturing confluent cultures of cells with $20 \mu 1$ pipette tips. These wounds were then covered with Matrigel to mimic cell communication with the microenvironment. Areas of wounds were marked and photographed every $15 \mathrm{~min}$ for $24 \mathrm{~h}$ using a Carl Zeiss Axio Observer Z1 inverted microscope (Carl Zeiss, Thornwood, NY). The distance of cell movement toward the wound space after $24 \mathrm{~h}$ was measured using live cell imaging software.

In vitro invasion assay. The invasiveness of cholangiocarcinoma cells was assayed in a 24-well Biocoat Matrigel invasion chamber ( $8 \mu \mathrm{m}$; Becton-Dickinson, Bioscience, Bedford, MA, USA). The upper chamber was seeded with 50,000 cells, and the lower chamber contained $1 \%$ FBS. After $24 \mathrm{~h}$ of incubation, the invading cells at the lower surface of the Matrigel-coated membrane were fixed with $25 \%$ methanol, stained with crystal violet (Sigma Chemical Co., St. Louis, MO) and counted in five random 400X power fields under a light microscope.

Migration assay. The migration assay was performed using 24-well transwell chambers ( $8 \mathrm{~mm}$; Becton-Dickinson). A total of 20,000 cells were seeded into the transwell chamber and allowed to migrate for $24 \mathrm{~h}$. The migrating cells at the lower surface of the transwell chamber were stained with crystal violet and counted in five random $400 \mathrm{X}$ power fields under a light microscope.

Inhibition of CD24 expression in RMCCA1 cells. Inhibition of CD24 expression was carried out using siRNA targeting CD24 (Santa Cruz Biotechnology, Inc., Santa Cruz, CA) according to the manufacturer's protocol. Briefly, $2 \times 10^{5}$ cells were plated in 6 -well tissue culture plates in antibiotic-free normal growth medium until the cells were $60-80 \%$ confluent. Cells were then incubated with a transfection reagent mixture (Ham's F-12, Lipofectamine (Invitrogen, Carlsbad, CA) and the CD24directed oligonucleotides (20-80 pmols) ) for $6 \mathrm{~h}$ at $37^{\circ} \mathrm{C}$. The transfection mixture was then replaced with normal growth medium, and the cells were incubated for $24 \mathrm{~h}$. The medium was again replaced with fresh normal growth medium, and the cells were incubated for another $24 \mathrm{~h}$ before harvesting for experimental use. Block-iT Fluorescent Control (Invitrogen, Carlsbad, CA) was used to confirm the transfection efficiency.

Detection of matrix metalloproteinase (MMP)-9. Gelatin zymography was used to detect the expression of MMP-9 in $\mathrm{CD}_{24}{ }^{+}$and CD24- cells. Six million cells were plated in normal medium for $24 \mathrm{~h}$ of culture, and the medium was then replaced with serum-free medium. After another $24 \mathrm{~h}$ of culture, the conditioned medium was collected, mixed with non-reducing sample buffer (2\% SDS, $10 \%$ glycerol, $62.5 \mathrm{mM}$ 

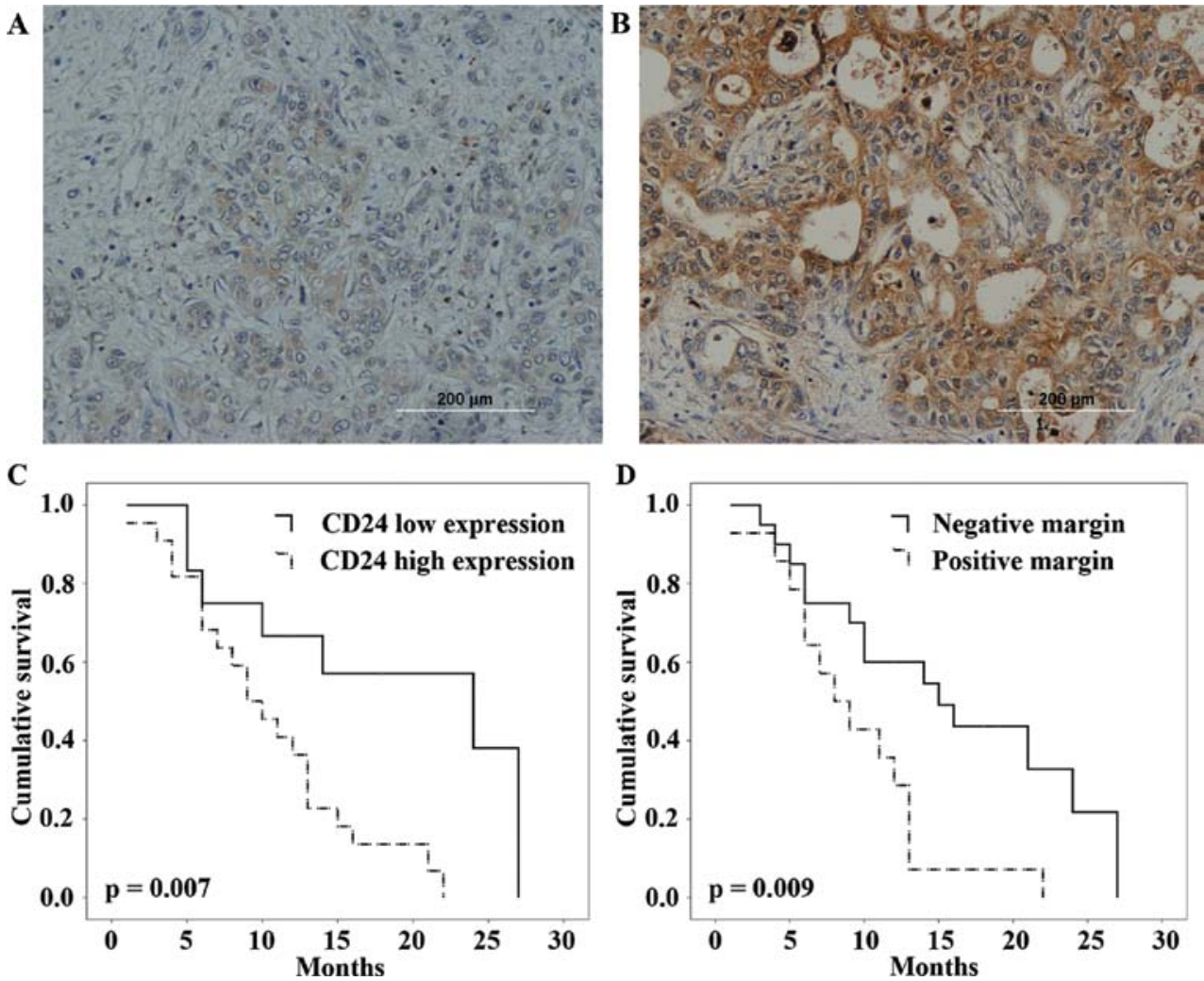

Figure 1. Representative immunohistochemical staining for CD24 in cholangiocarcinoma samples. (A) Cholangiocarcinoma cells with low expression of CD24. (B) Cholangiocarcinoma cells with high expression of CD24. Positive staining was observed in the cytoplasm of cholangiocarcinoma cells (magnification x200). (C) Survival curves of patients with high and low CD24 expression ( $\mathrm{p}=0.007)$. (D) Survival curves of patients with positive and negative surgical margins $(\mathrm{p}=0.009)$.

Tris-Cl, pH 6.8, and $0.01 \%$ bromophenol blue) and separated on a $7.5 \%$ polyacrylamide gel containing $0.1 \%$ gelatin. After electrophoresis, the gel was soaked twice in $2.5 \%$ Triton X-100 in $50 \mathrm{mM}$ Tris- $\mathrm{Cl}, \mathrm{pH} 7.5$, for $30 \mathrm{~min}$ and then incubated with $0.15 \mathrm{M} \mathrm{NaCl}, 10 \mathrm{mM} \mathrm{CaCl}_{2}$ in $50 \mathrm{mM}$ Tris-Cl, $\mathrm{pH} 7.5$, for $18 \mathrm{~h}$ at $37^{\circ} \mathrm{C}$. The gel was then stained with $0.25 \%(\mathrm{w} / \mathrm{v})$ Coomassie blue R250 and destained for $1 \mathrm{~h}$.

Detection of matrix metalloproteinase (MMP)-7. The Human Total MMP-7 Immunoassay kit (R\&D Systems, Minneapolis, $\mathrm{MN}$ ) was used to detect the expression of MMP-7 in CD24 ${ }^{+}$ and CD24 cells. Six million cells were plated in culture dishes. After $24 \mathrm{~h}$ of culture, the normal medium was replaced with serum-free medium, and the cells were cultured for another $24 \mathrm{~h}$. The conditioned medium was collected and measured for the expression of MMP-7 following the manufacturer's protocol.

Statistical analysis. Statistical analysis of the association between clinicopathological data and the expression of CD24 was performed using the $\chi^{2}$ test or Fisher's exact test. The Kaplan-Meier method was used to estimate survival as a function of time, and the survival differences were analyzed by log-rank test. The Cox regression model was used for multivariate analysis of prognostic factors. The cell proliferation and invasion assays were performed in triplicate, and each result was reported as the mean with standard deviation. Data were compared using the Student's t-test. A p $<0.05$ was considered statistically significant.

\section{Results}

Expression ofCD24 inparaffin-embedded cholangiocarcinoma samples. Upon immunohistochemical analysis of non-cancer bile duct tissues, CD24 expression was not detected. In cancer tissues, specific CD24 signals were localized mainly in the cell membrane and cytoplasm of cholangiocarcinoma cells (Fig. 1A and B). We found that all cholangiocarcinoma samples (34/34) exhibited CD24 expression ( $>5 \%$ of cell staining). Applying the criteria for intensity of immunohistochemical staining for CD24, high expression (>50\% of cell staining) of CD24 was noted in $64.7 \%(22 / 34)$ of cholangiocarcinoma samples. With respect to clinicopathological variables, we found that nodal metastasis $(\mathrm{p}=0.003)$ and a positive surgical margin ( $\mathrm{p}=0.005)$ were more frequent in the CD24 high expression group compared with the CD24 low expression group (Table I). Univariate analysis revealed that the median survival time was 9 months in patients with high CD24 expression, whereas the survival time was 24 months in patients with low CD24 expression $(p=0.007)$. We also found that the median survival time in patients with a negative surgical margin was significantly better than in patients with a positive surgical margin (15 months vs. 8 months; $\mathrm{p}=0.009$ ) (Fig. 1C and D). In a multivariate Cox regression analysis, $\mathrm{CD} 24$ was indicated as an independent risk factor for survival ( $\mathrm{p}=0.040$; Table II).

\section{In vitro study}

Enrichment of $C D 24^{+}$RMCCA1 cells. The results from the immunohistochemical staining indicated that high expression 
Table I. Relationship between clinicopathological features and CD24 expression in cholangiocarcinoma samples.

\begin{tabular}{|c|c|c|c|}
\hline \multirow[b]{2}{*}{ Variable } & \multicolumn{3}{|c|}{ CD24 expression } \\
\hline & $\begin{array}{c}\text { Total } \\
(\mathrm{n}=34)\end{array}$ & Low High & p-value \\
\hline
\end{tabular}

$$
\begin{gathered}
\text { Age } \\
<60 \\
>60
\end{gathered}
$$$$
18
$$

$8 \quad 10$

$\begin{array}{lll}16 & 4 & 12\end{array}$

0.477

Sex

$\begin{array}{lrrrr}\text { Male } & 22 & 9 & 13 & 0.705 \\ \text { Female } & 12 & 3 & 9 & \end{array}$

Tumor differentiation

$\begin{array}{lrrrr}\text { Well } & 17 & 9 & 8 & 0.071 \\ \text { Moderate/poor } & 17 & 3 & 14 & \end{array}$

Node

$\begin{array}{lrrrr}\text { Negative } & 16 & 10 & 6 & 0.003^{\mathrm{a}} \\ \text { Positive } & 18 & 2 & 16 & \end{array}$

Distant metastasis

$\begin{array}{lrrrr}\text { Negative } & 24 & 11 & 13 & 0.061 \\ \text { Positive } & 10 & 1 & 9 & \end{array}$

Surgical resection margin ${ }^{\mathrm{b}}$

\begin{tabular}{lrrrr} 
R0 & 20 & 11 & 9 & $0.005^{\mathrm{a}}$ \\
R1, R2 & 14 & 1 & 13 & \\
\hline
\end{tabular}

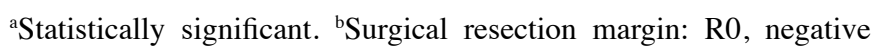
resection margin; R1, microscopic positive resection margin; and R2, macroscopic positive resection margin.

of CD24 was associated with poor prognosis in cholangiocarcinoma patients. Therefore, we performed in vitro studies to identify the role of CD24 in cholangiocarcinoma cell progression. We isolated CD24+ and CD24- cells from the RMCCA1 cholangiocarcinoma cell line using magnetic cell sorting technology. The percentage of CD24 $4^{+}$cells in CD24-enriched cells $\left(\mathrm{CD} 24^{+}\right)$was $91.9 \%$, and the percentage of $\mathrm{CD} 24^{+}$cells in the CD24-depleted cells (CD24) was 11.5\%; the percentage of $\mathrm{CD} 24^{+}$cells in naïve RMCCA1 cells was $28.9 \%$ (Fig. 2A). As revealed by morphological studies, no differences in cell morphology were observed between $\mathrm{CD} 24^{+}$and $\mathrm{CD} 24^{-}$ RMCCA1 cells (Fig. 2B).

Proliferation of $\mathrm{CD}_{2} 4^{+}$and $\mathrm{CD} 24^{-}$cholangiocarcinoma cells. CD24 expression has been shown to stimulate cancer cell proliferation. We therefore investigated the rate of cell proliferation in $\mathrm{CD}_{2} 4^{+}$and CD24 ${ }^{-}$MCCA1 cells cultured for 0-9 days. We found that there was no statistically significant difference in cell proliferation between the CD24+ and CD24cell populations (Fig. 3A).

Chemoresistance of $C D 24^{+}$and CD24-RMCCA1 cells. Previous studies have suggested that $\mathrm{CD} 24$ expression is responsible for the lack of response to chemotherapy (10). Here, we investigated the response of $\mathrm{CD} 24^{+}$and $\mathrm{CD} 24^{-}$cells to cisplatin, an anticancer agent that is used to treat cholangiocarcinoma patients. The results demonstrated that $\mathrm{CD} 24^{+}$cells had an $\mathrm{IC}_{50} 1.7$-fold higher $(7.5 \mu \mathrm{g} / \mathrm{ml})$ than the corresponding $\mathrm{IC}_{50}$ value in $\mathrm{CD} 24$ cells $(4.5 \mu \mathrm{g} / \mathrm{ml})$ in response to cisplatin (Fig. 3B).

Cell adhesion assay in $\mathrm{CD}^{2} 4^{+}$and CD24- RMCCA1 cells. Previous work has demonstrated that CD24 enhances cell adhesion (6). Therefore, we investigated the role of CD24 in cell adhesion in $\mathrm{CD} 24^{+}$and CD24- RMCCA1 cells. Our data demonstrated that $\mathrm{CD} 24^{+}$cells adhered to Matrigel coated plates significantly better than $\mathrm{CD}^{-} 4^{-}$cells ( $\mathrm{p}=0.004$ ). However, cell adhesion to non-coated plates was not significantly different between CD24+ and CD24- cells (Fig. 4A). This finding suggests that CD24 enhances cell adhesion by allowing cells to adhere to extracellular matrix proteins.

Cell motility assay in $C D 24^{+}$and CD24- RMCCA1 cells. A previous study reported that $\mathrm{CD} 24^{+}$cells are more motile than CD24 cells (13). In our work, time-lapse live cell imaging was performed to investigate cell migrating across a wound gap. The motility of $\mathrm{CD} 24^{+}$cells to cover the wound was significantly greater than that of the CD24- cells $(\mathrm{p}<0.001)$ (Fig. 4B).

Table II. Survival analysis based on clinicopathological parameters using Cox's multivariate model.

\begin{tabular}{lcccc}
\hline & & & \multicolumn{2}{c}{$95 \%$ confidence interval } \\
\cline { 4 - 5 } Variables & p-value & Hazard ratio & Lower limit & Upper limit \\
\hline Tumor differentiation & 0.26 & 0.58 & 0.22 & 1.51 \\
Lymph node metastasis & 0.49 & 0.66 & 0.19 & 2.02 \\
Distant metastasis & 0.45 & 0.64 & 0.20 & 2.06 \\
Surgical resection status & 0.11 & 2.66 & 0.80 & 8.78 \\
CD24 expression & $0.04^{\mathrm{a}}$ & 4.31 & 1.06 & 17.48 \\
\hline
\end{tabular}

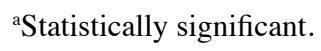


A

RMCCAl

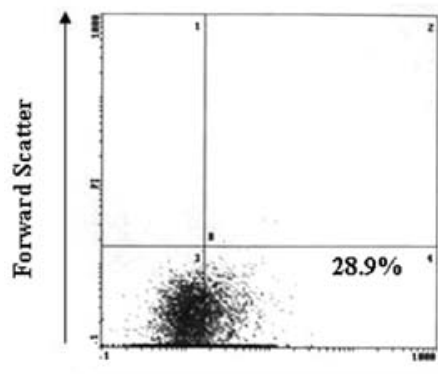

B

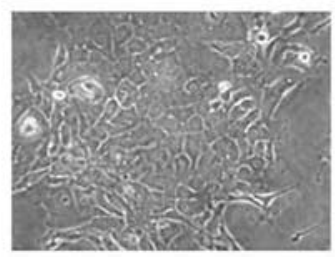

$\mathrm{CD} 24+$

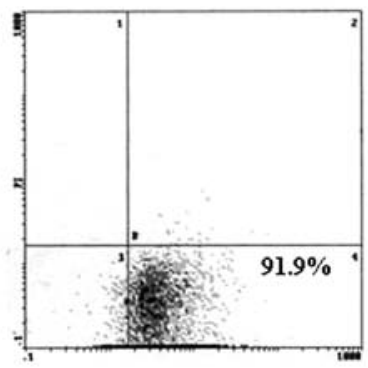

CD24-FITC

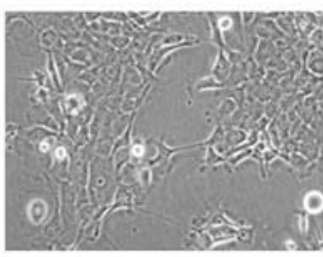

CD24-
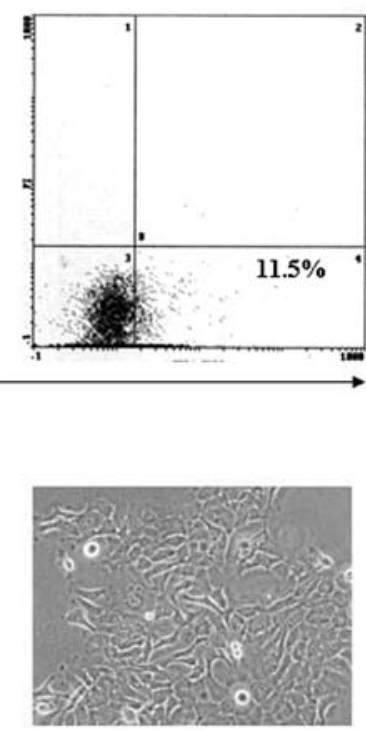

Figure 2. Analysis of purity of sorted populations. CD24 ${ }^{+}$and CD24- cell fractions were separated from RMCCA1 cells using MACS. Sorted CD24 ${ }^{+}$and CD24- cell subsets were analyzed by flow cytometry to evaluate the efficiency of sorting. (A) Percentage of CD24 $4^{+}$cells in RMCCA1, the enriched (CD24+) and depleted (CD24) fractions. (B) Cell morphology of CD24+ and CD24- fractions.

A

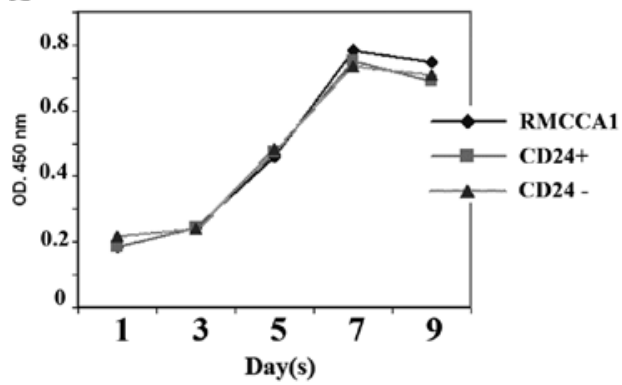

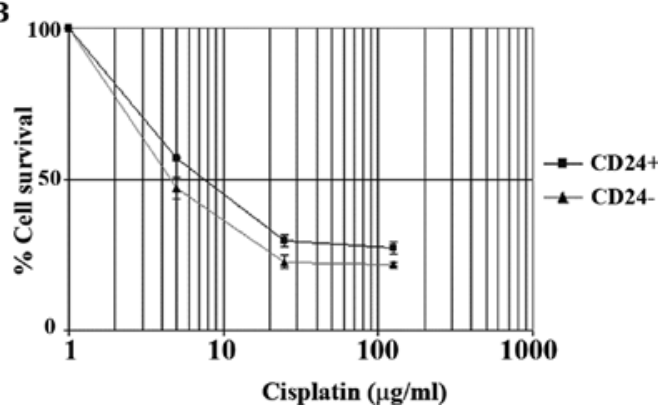

Figure 3. (A) MACS-sorted CD24+ and CD24- cells exhibit a similar degree of cell proliferation. Cells were isolated by MACS and subjected to in vitro assays for cell

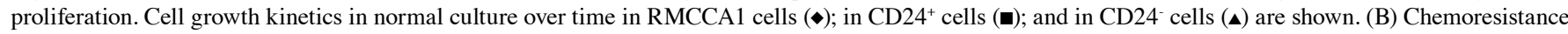
of $\mathrm{CD} 24^{+}$and $\mathrm{CD} 24^{-}$cells to cisplatin. The $\mathrm{IC}_{50}$ represents the drug concentration that resulted in a $50 \%$ decrease in cell survival.

A

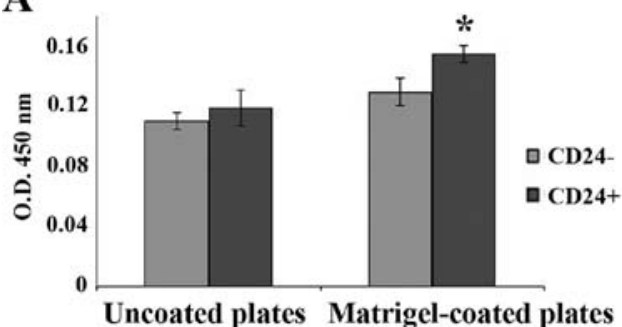

Figure 4. (A) $\mathrm{CD} 24^{+}$cells demonstrate enhanced cell adhesion to Matrigel. Cells were allowed to adhere to 96 -well tissue culture plates uncoated or pre-coated with Matrigel. Data are presented as the mean \pm SD. "Significant difference in cell number when compared to the CD24- cell subset $(\mathrm{p}=0.004)$

(B) $\mathrm{CD} 24^{+}$cells demonstrate enhanced cell motility towards Matrigel. Wounds were made in confluent CD24+ and CD24- cells, and the cells were allowed to heal by migration. Wounds were marked, and distance of cell movement into the wound was monitored by time-lapse live cell imaging. Data are presented as the mean $\pm \mathrm{SD}$. ${ }^{* *}$ Significant difference in cell motility when compared to the CD24- cell subset $(\mathrm{p}<0.001)$.
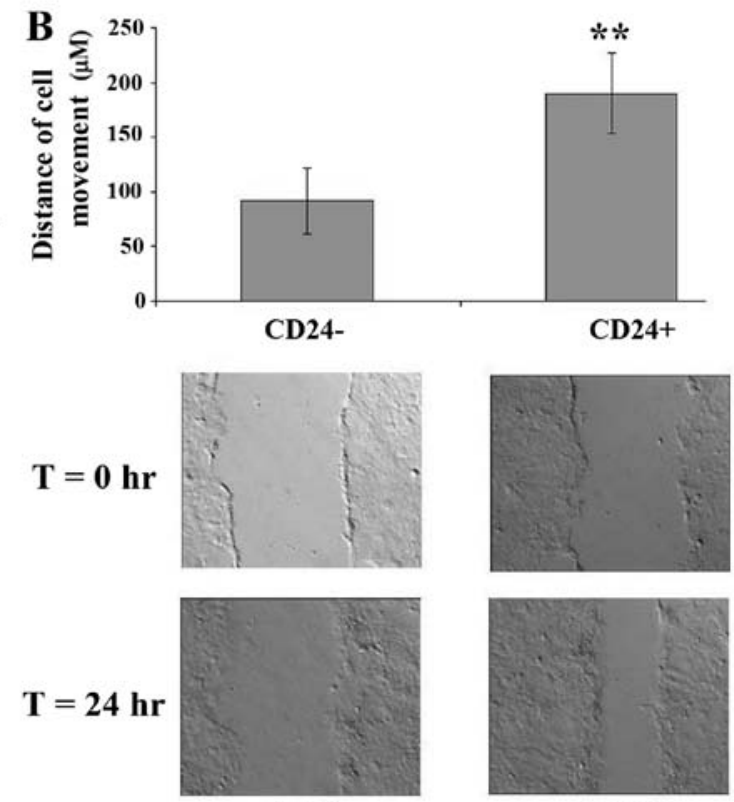

CD24-

CD24+ 


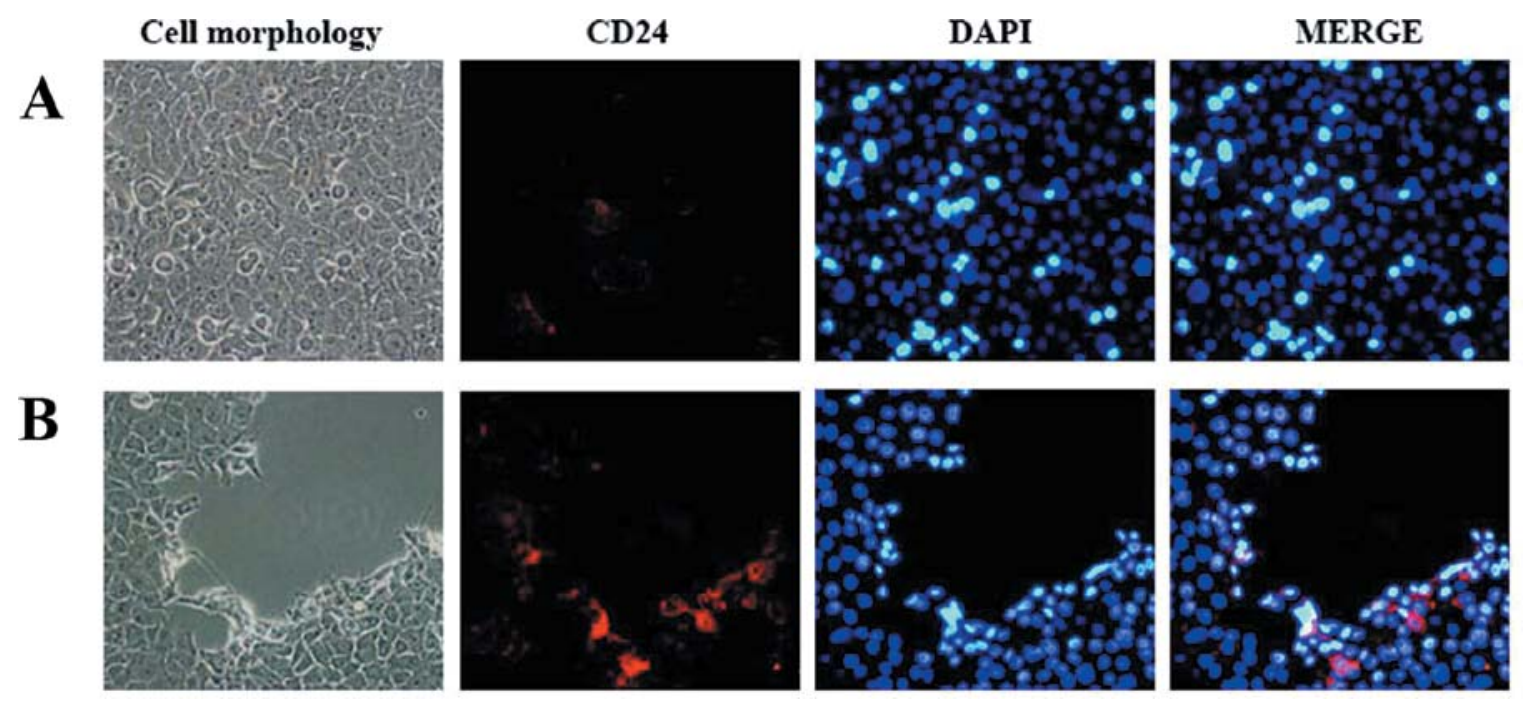

Figure 5. Wounds were created in confluent RMCCA1 cells, and immunofluorescence staining was performed to compare (A) CD24 expression in cells distant from and (B) cells at the wound edge. Cells at the wound edge expressed high levels of CD24 compared to the cells distant from the wound.
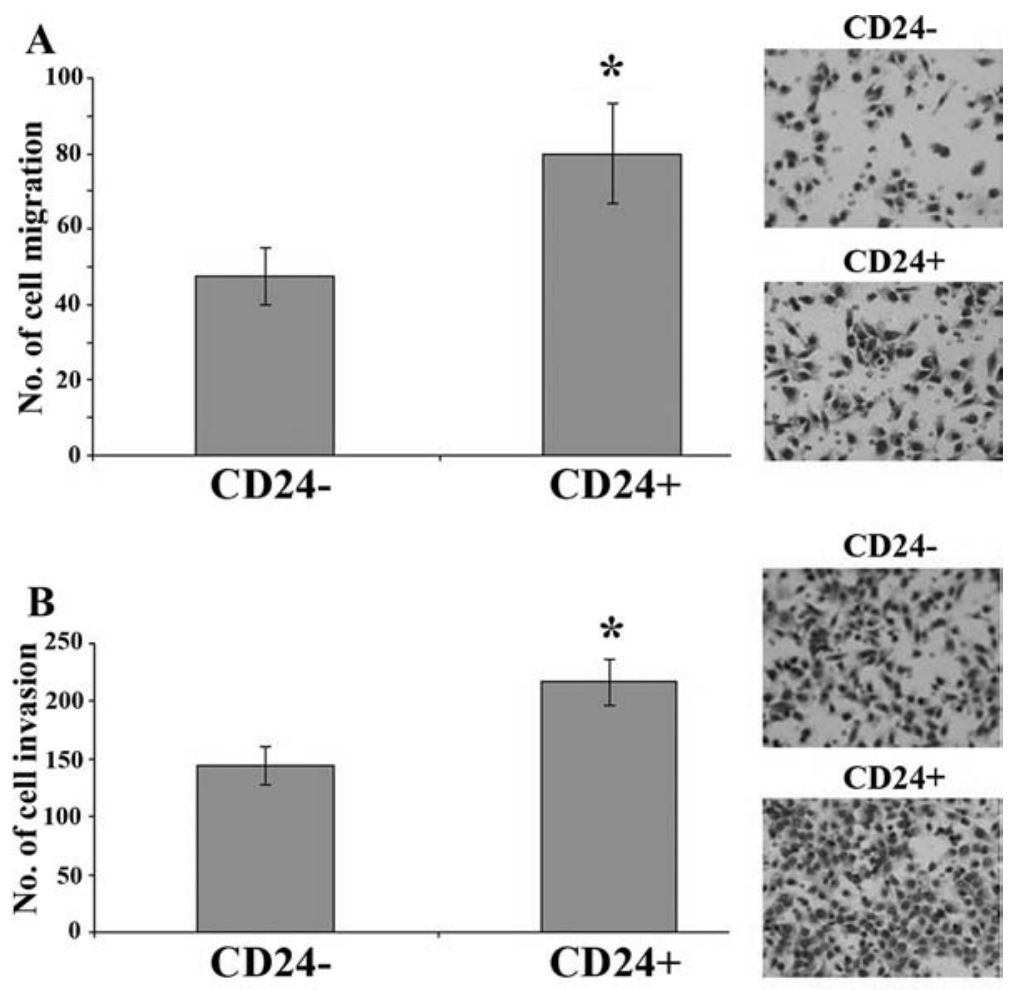

Figure 6. (A) $\mathrm{CD} 24^{+}$cells demonstrate enhanced cell migration and (B) cell invasion in vitro. The migration and invasion capabilities of CD24- and CD24 cells isolated from the RMCCA1 cell line were assessed. Transwells were uncoated (migration assays) or pre-coated with Matrigel (invasion assays), and cells were allowed to migrate or invade for $24 \mathrm{~h}$ and counted. Data are presented as the mean $\pm \mathrm{SD}$. "Significant difference in cell number when compared to the CD24- cell subset $(\mathrm{p}<0.001)$.

We also performed a wound-healing assay in naïve RMCCA1 cells. Immunofluorescence staining with an antiCD24 antibody demonstrated that the cells at the wound edge expressed higher levels of CD24 than the cells distant from the sight of the wound (Fig. 5).

Cell migration and invasionassay in $C D 24^{+}$and CD24 RMCCAI cells. Previous studies have shown that CD24 expression is associated with tumor aggressiveness, as demonstrated by increased metastasis and invasiveness $(13,14)$. In the present study, transwell migration and invasion assays were performed to assess the metastatic potential of the cell populations. The $\mathrm{CD} 24^{+}$cell subset showed a significantly greater ability to migrate towards serum (Fig. 6A) and invade through Matrigel (Fig. 6B) than the CD24- cell subset ( $<<0.001)$.

Detection of MMP-9 and MMP-7 in the conditioned medium of $C D 24^{+}$and CD24- RMCCA1 cells. Matrix metalloproteinases 


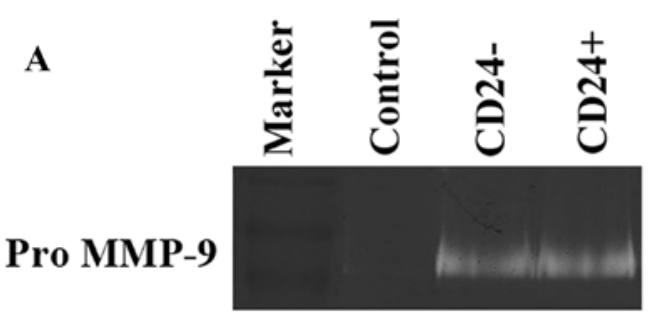

B

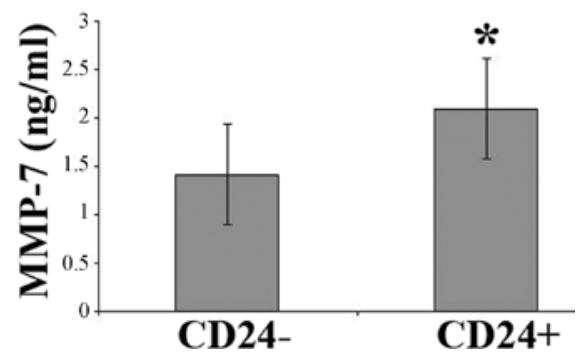

Figure 7. (A) MMP-9 activity in $\mathrm{CD} 24^{+}$and $\mathrm{CD} 24^{-}$cell populations; medium with $1 \%$ FBS (positive control) and conditioned medium from $\mathrm{CD} 24^{+}$and CD24- cell populations were subjected to gelatin zymography. Matrix metalloproteinase activity is shown as a clear band on the gel. (B) MMP-7 levels in $\mathrm{CD} 24^{+}$and CD24- cell populations. The secreted level of MMP-7 in the conditioned medium collected from both CD24+ and CD24- cell populations was assessed by the Human Total MMP-7 ELISA kit. Data are presented as the mean \pm SD. "Significant difference in cell number when compared to the $\mathrm{CD} 24$ cell subset $(\mathrm{p}=0.01)$.
(MMPs) have long been associated with cancer-cell invasion and metastasis via the cleavage of basement membrane and extracellular matrix proteins to allow for cells to invade tissues and spread throughout the body $(15,16)$. Here, we investigated the expression of MMP-9 by harvesting conditioned medium from both $\mathrm{CD}_{24}{ }^{+}$and $\mathrm{CD} 24^{-}$cell populations and performing gelatin zymography. We found that the conditioned medium from both cell populations expressed similar levels of MMP-9 as observed by the similar intensity of the clear band at $\sim 92 \mathrm{kDa}$, which corresponds to the known size of pro-MMP-9 (Fig. 7A).

We also examined the secreted level of MMP-7 in the conditioned medium of both cell populations by enzymelinked immunosorbent assay (ELISA). The results showed that MMP-7 levels were significantly higher in the conditioned medium of $\mathrm{CD} 24^{+}$cells than in the conditioned medium of CD24- cells ( $\mathrm{p}=0.01)$ (Fig. 7B).

Inhibition of CD24 expression decreased cell invasion. We further sought to confirm the correlation between CD24 expression and cell invasion by silencing CD24 expression in RMCCA1 cells. As demonstrated by immunofluorescence, RMCCA1 cells that were treated with CD24 siRNA showed a reduced CD24 signal when compared with the cells treated with control siRNA (Fig. 8A). The results of the invasion assay showed that the cells

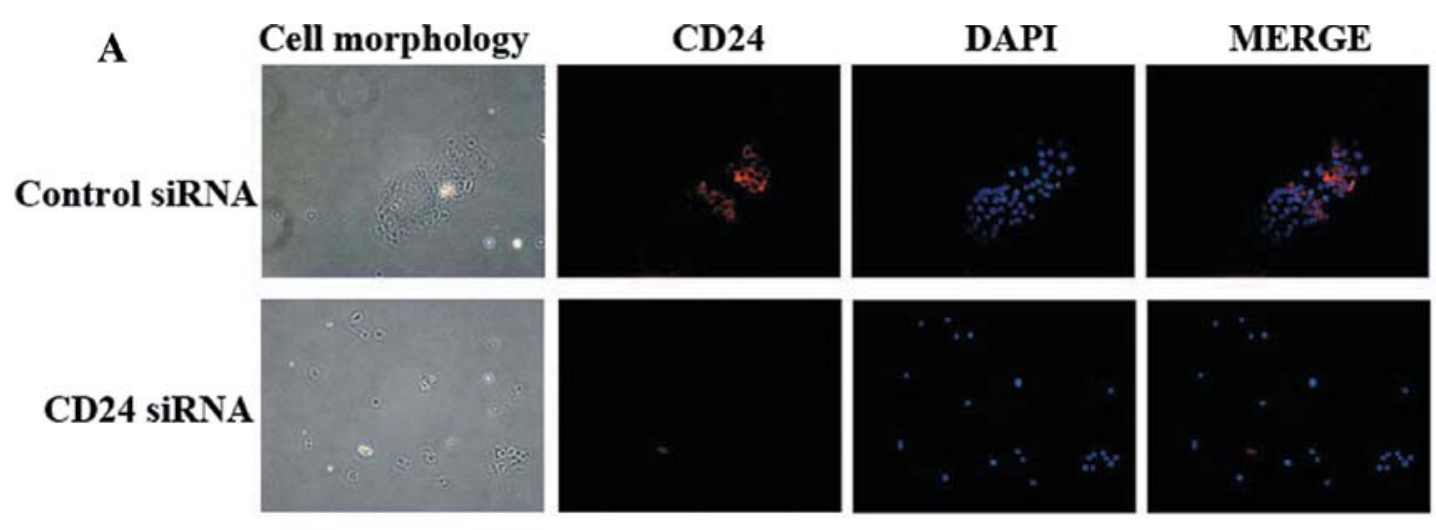

B

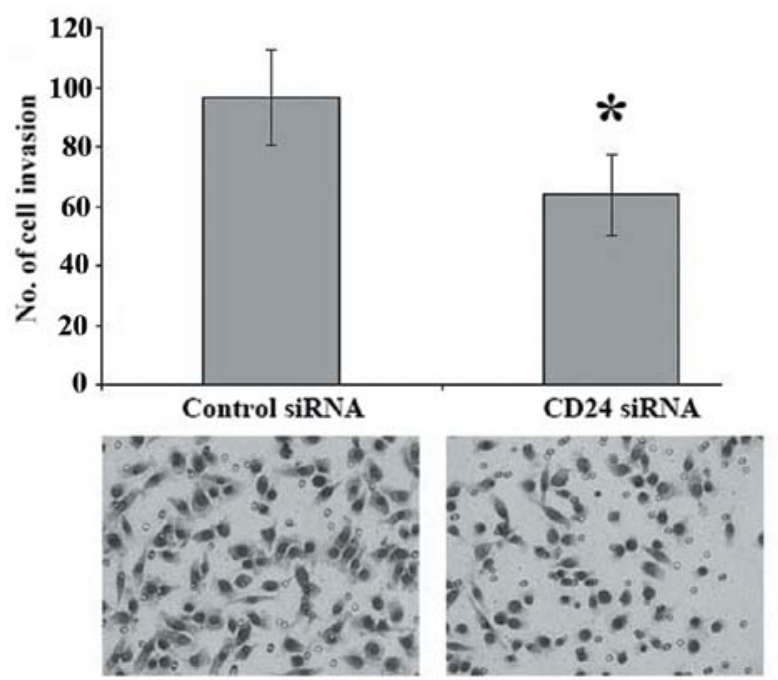

Figure 8. Inhibition of CD24 expression results in decreased cell invasion. (A) Immunofluorescence staining in RMCCA1 cells and CD24 siRNA-treated RMCCA1 cells using an anti-CD24 antibody. (B) Cell invasion capabilities of RMCCA1 cells treated with control or CD24 siRNA. Data are presented as the mean \pm SD. "Significant difference in cell number when compared to the CD24- cell subset $(\mathrm{p}<0.001)$. 
that were treated with CD24 siRNA had a significantly diminished capacity to invade through the Matrigel when compared with the cells treated with control siRNA ( $<<0.001)$ (Fig. 8B).

\section{Discussion}

In this study, we demonstrated that $64.7 \%$ of the cholangiocarcinoma samples exhibited high expression of CD24. Moreover, we showed that high expression of CD24 correlated with lymph node metastasis and overall survival of the cholangiocarcinoma patients. These findings are consistent with a previous study, which demonstrated that cholangiocarcinomas highly expressing CD24 tended to be associated with higher incidences of metastasis and worse prognoses $(10,11)$. In addition, many studies have shown that CD24 expression correlates with the aggressiveness of other types of cancer, including pancreatic cancer (17) and hepatocellular carcinoma (18). Taken together, our data indicate that $\mathrm{CD} 24$ is involved in the progression and malignant behavior of cholangiocarcinoma.

To understand the role of CD24 in cholangiocarcinoma cells, we compared the cell proliferation, chemoresistance and invasion potential of isolated CD $24^{+}$and CD24- RMCCA1 cholangiocarcinoma cell populations. We found no difference in cell proliferation between $\mathrm{CD} 24^{+}$and CD24- RMCCA1 cells. However, the chemoresistance assay demonstrated that CD24+ RMCCA1 cells exhibited more resistance to cisplatin than CD24- RMCCA1 cells. This result is also consistent with recent evidence showing that the mechanism of drug resistance in colon and pancreatic cancer cells (19) is through the expression of CD24. Our studies therefore indicate that expression of CD24 may be the key mechanism governing the chemoresistant phenotype of cholangiocarcinoma. Further studies are required to identify the role of CD24 in cholangiocarcinoma patients receiving chemotherapy.

Our results are consistent with previous work demonstrating that downregulation of CD24 in colorectal and pancreatic cancer by RNA interference or by using anti-CD24 monoclonal antibodies significantly inhibit cell invasion (19). We demonstrated that inhibiting CD24 by siRNA reduced the invasive capacity of RMCCA1 cells, indicating that CD24 plays an important role in cholangiocarcinoma progression via its potential to promote invasion. The requirements for tumor cell invasion consist of the following: i) adhesion of tumor cells to the extracellular matrix $(E C M)$, ii) migration of tumor cells through the ECM and iii) degradation of the ECM by cellular proteases (20). The ability of $\mathrm{CD} 24^{+}$cells to invade has generally been reported to be due to the adhesion between CD24 and extracellular molecules. A previous study demonstrated that the $\mathrm{CD} 24^{\text {high }} \mathrm{MCF}-7$ cells were more adhesive than the CD2 $4^{\text {low }}$ cells (21). In our study, we also observed that $\mathrm{CD} 24^{+}$cells had a greater ability to adhere to Matrigel than CD24- cells. However, there was no significant difference between $\mathrm{CD} 24^{+}$and $\mathrm{CD} 24^{-}$cells to adhere to noncoated culture plates. It is likely that the increased adhesive ability of CD24+ cells was due to the binding of CD24 with the extracellular molecules in the Matrigel. Taken together, these results suggest that CD24 enhances the adhesiveness of cholangiocarcinoma cells.

We investigated the capability of cells to migrate by using a wound-healing assay. After a wound is created in cultured confluent cells, cells at the edge of the wound will migrate across the wound gap. We found that the motility of CD24 $4^{+}$cells was higher than that of CD24- cells. This finding is consistent with a previous study in which forced expression of CD24 in HCT116 human colon cancer cells significantly increased cell migration in wound-healing assays (22). We also performed a wound-healing assay in naïve RMCCA1 cells and found that RMCCA1 cells at the wound edge expressed higher levels of CD24, suggesting that $\mathrm{CD} 24^{-}$cells can give rise to $\mathrm{CD} 24^{+}$cells that have a greater ability to migrate. These data indicate that cholangiocarcinoma cells can change their protein expression in order to acquire the capacity to invade.

We further examined the expression of proteases in $\mathrm{CD} 24^{+}$ and CD24- cells. We demonstrated for the first time that MMP-7 was highly expressed in $\mathrm{CD} 24^{+}$cholangiocarcinoma cells. Previous studies have demonstrated that MMP-7 plays a key role in the mechanism of cancer invasion via proteolytic cleavage of the extracellular matrix tissues (22). MMP-7 has also been shown to activate other MMPs, such as pro-MMP-2 and pro-MMP-9, and to inhibit E-cadherin function by inducing ectodomain shedding of E-cadherin $(23,24)$. The results of several recent studies have indicated that MMP-7 is overexpressed in a variety of epithelial cancers, including esophagus, colon, pancreas, and cholangiocarcinoma, and is associated with cancer progression (25-28). Thus, the invasive capacity of $\mathrm{CD} 24^{+}$cells not only depends on their adherent capabilities but also on increased MMP-7 expression.

In conclusion, CD24 is highly expressed in cholangiocarcinoma, and it significantly correlates with lymph node metastasis. CD24 should therefore be used as a potential prognostic factor and target for treatment of cholangiocarcinoma.

\section{References}

1. Sripa B and Pairojkul C: Cholangiocarcinoma: lessons from Thailand. Curr Opin Gastroenterol 24: 349-356, 2008.

2. Akoad M and Jenkins R: Proximal biliary malignancy. Surg Clin North Am 88: 1409-1428, x-xi, 2008.

3. Sano T, Shimada K, Sakamoto Y, Ojima H, Esaki M and Kosuge T: Prognosis of perihilar cholangiocarcinoma: hilar bile duct cancer versus intrahepatic cholangiocarcinoma involving the hepatic hilus. Ann Surg Oncol 15: 590-599, 2008.

4. Shimada K, Sano T, Sakamoto Y, Esaki M, Kosuge T and Ojima H: Clinical impact of the surgical margin status in hepatectomy for solitary mass-forming type intrahepatic cholangiocarcinoma without lymph node metastases. J Surg Oncol 96: 160-165, 2007.

5. Tucek S, Tomasek J, Halamkova J, et al: Bile duct malignancies. Klin Onkol 23: 231-241, 2010.

6. Kristiansen G, Sammar M and Altevogt P: Tumour biological aspects of CD24, a mucin-like adhesion molecule. J Mol Histol 35: 255-262, 2004

7. Redondo P, Garcia-Foncillas J, Okroujnov I, De Felipe I and Quintanilla E: CD24 expression on human keratinocytes. Exp Dermatol 7: 175-178, 1998.

8. Lee HJ, Choe G, Jheon S, Sung SW, Lee CT and Chung JH: $\mathrm{CD} 24$, a novel cancer biomarker, predicting disease-free survival of non-small cell lung carcinomas: a retrospective study of prognostic factor analysis from the viewpoint of forthcoming (seventh) new TNM classification. J Thorac Oncol 5: 649-657, 2010.

9. Lee JH, Kim SH, Lee ES and Kim YS: CD24 overexpression in cancer development and progression: a meta-analysis. Oncol Rep 22: 1149-1156, 2009.

10. Agrawal S, Kuvshinoff BW, Khoury T, et al: CD24 expression is an independent prognostic marker in cholangiocarcinoma. J Gastrointest Surg 11: 445-451, 2007.

11. Su MC, Hsu C, Kao HL and Jeng YM: CD24 expression is a prognostic factor in intrahepatic cholangiocarcinoma. Cancer Lett 235: 34-39, 2006. 
12. Rattanasinganchan $\mathrm{P}$, Leelawat $\mathrm{K}$, Treepongkaruna SA, et al: Establishment and characterization of a cholangiocarcinoma cel line (RMCCA-1) from a Thai patient. World J Gastroenterol 12: 6500-6506, 2006

13. Baumann P, Cremers N, Kroese F, et al: CD24 expression causes the acquisition of multiple cellular properties associated with tumor growth and metastasis. Cancer Res 65: 10783-10793, 2005

14. Senner V, Sturm A, Baur I, Schrell UH, Distel L and Paulus W: CD24 promotes invasion of glioma cells in vivo. J Neuropathol Exp Neurol 58: 795-802, 1999.

15. Egeblad M and Werb Z: New functions for the matrix metalloproteinases in cancer progression. Nat Rev Cancer 2: 161-174, 2002.

16. Sternlicht MD and Werb Z: How matrix metalloproteinases regulate cell behavior. Annu Rev Cell Dev Biol 17: 463-516, 2001.

17. Ikenaga N, Ohuchida K, Mizumoto K, et al: Characterization of CD24 expression in intraductal papillary mucinous neoplasms and ductal carcinoma of the pancreas. Hum Pathol 41: 1466-1474, 2010.

18. Yang XR, Xu Y, Yu B, et al: CD24 is a novel predictor for poor prognosis of hepatocellular carcinoma after surgery. Clin Cancer Res 15: 5518-5527, 2009.

19. Sagiv E, Starr A, Rozovski U, et al: Targeting CD24 for treatment of colorectal and pancreatic cancer by monoclonal antibodies or small interfering RNA. Cancer Res 68: 2803-2812, 2008.

20. Yilmaz M and Christofori G: Mechanisms of motility in metastasizing cells. Mol Cancer Res 8: 629-642, 2010.

21. Kim HJ, Kim JB, Lee KM, et al: Isolation of CD24(high) and CD24(low/-) cells from MCF-7: CD24 expression is positively related with proliferation, adhesion and invasion in MCF-7. Cancer Lett 258: 98-108, 2007.
22. Ahmed MA, Jackson D, Seth R, et al: CD24 is upregulated in inflammatory bowel disease and stimulates cell motility and colony formation. Inflamm Bowel Dis 16: 795-803, 2010.

23. Ii M, Yamamoto H, Adachi Y, Maruyama Y and Shinomura Y: Role of matrix metalloproteinase-7 (matrilysin) in human cancer invasion, apoptosis, growth, and angiogenesis. Exp Biol Med (Maywood) 231: 20-27, 2006.

24. Noe V, Fingleton B, Jacobs K, et al: Release of an invasion promoter E-cadherin fragment by matrilysin and stromelysin-1.J Cell Sci 114: 111-118, 2001.

25. Yamashita K, Mori M, Shiraishi T, Shibuta K and Sugimachi K: Clinical significance of matrix metalloproteinase-7 expression in esophageal carcinoma. Clin Cancer Res 6: 1169-1174, 2000.

26. Jones LE, Humphreys MJ, Campbell F, Neoptolemos JP and Boyd MT: Comprehensive analysis of matrix metalloproteinase and tissue inhibitor expression in pancreatic cancer: increased expression of matrix metalloproteinase-7 predicts poor survival. Clin Cancer Res 10: 2832-2845, 2004.

27. Itatsu K, Zen Y, Yamaguchi J, et al: Expression of matrix metalloproteinase 7 is an unfavorable postoperative prognostic factor in cholangiocarcinoma of the perihilar, hilar, and extrahepatic bile ducts. Hum Pathol 39: 710-719, 2008.

28. Leelawat K, Narong S, Wannaprasert J and Ratanashu-ek T: Prospective study of MMP7 serum levels in the diagnosis of cholangiocarcinoma. World J Gastroenterol 16: 4697-4703, 2010. 